\title{
Time-Reversal UWB Wireless Communication-Based Train Control in Tunnel
}

\author{
Hassane Saghir*†, Marc Heddebaut ${ }^{\dagger}$, Fouzia Elbahhar ${ }^{\dagger}$, Atika Rivenq*, Jean Michel Rouvaen* \\ * IEMN-DOAE, University of Valenciennes, Valenciennes, France. \\ Email: \{firstname.lastname\}@univ-valenciennes.fr \\ $\dagger$ INRETS-LEOST, French national institute for transport and safety research, Villeneuve d'Ascq, France. \\ Email: $\{$ firstname.lastname $\} @$ inrets.fr
}

\begin{abstract}
This paper reports an evaluation of UWB radio technology and Time-Reversal (TR) technique in tunnel environments for train-to-wayside communication. UWB technology has the potential to offer simultaneous ground-totrain communication, train location and obstacle detection in front of the trains. Time-Reversal channel pre-filtering facilitates signal detection and helps reduce interference. Thus, UWB-TR combination provides a challenging, economically sensible, as well as technically effective alternative solution to existing signaling technologies used in urban transport systems. This paper deals with deterministic channel modeling and its characterization in tunnel environment. It reports simulation performance evaluation of UWB-TR combinations in the developed channel model.
\end{abstract}

Index Terms-UWB, time-reversal, channel modeling, ray tracing, railway systems, CBTC, tunnel, propagation.

\section{INTRODUCTION}

For high-performance, economical and reliable transportation networks, optimization of railway infrastructure and train communication equipment are required. In order to provide wireless services and safety feeling anywhere at any time to passengers, advanced control and command systems integrate intelligent embedded radio frequency (RF) solutions in the railroad and aboard the trains. Through the computer-aided dispatching platforms, centralized traffic control centers (CTC) collect information about status, speed and location from the existing signal infrastructure. This information is used to send specific command instructions (voice and data) to each train and to display traffic information to passengers. This train-towayside data exchange is known by the acronym CBTC for Communication-Based Train Control.

Current requirements for CBTC, especially for driverless subway systems, necessitate real-time high datarate communication, typically several Mbps. To achieve this high transmission rate, current commercial solutions use radio modems working at high carrier frequencies, between 2 and $6 \mathrm{GHz}$ [1]. For some industrial suppliers [2], the radio modems are very close to the existing Wireless Local Area Network (WLAN) standards i.e. IEEE $802.11 \mathrm{a}, \mathrm{b} / \mathrm{g}$, but could migrate, when available, to the IEEE 802.11p standard (WAVE ${ }^{1}$ ) [3]. Some other

Manuscript received December 1, 2008; revised March 19, 2009; accepted March 23, 2009.

${ }^{1}$ Wireless Access in the Vehicular Environment industrial suppliers develop proprietary schemes CBTC radio systems [4] [5]. The whole transport network (infrastructure and trains) is built over a variety of environments (open-space, subway tunnels, viaducts, etc.). As a result, the characteristics of the train-to-wayside propagation channel will change while the train is moving. This impacts the communication system level of performance. Thus, the considered communication system has to match these environment changes. However, the tunnel is the most common environment for subway guided transport. Indeed, managing and optimizing the use of rail transport necessitates the exploitation of underground environments in order to decongest urban sites. In such environments, narrow bandwidth communication suffers from frequency and spatial selectivities. Due to signal attenuation along the tunnel according to the distance and the frequency used and due to heavy multi-path effect, RF transmission is subject to several problems. Previous studies have shown that free propagation in subway tunnels is effective at frequencies starting at $900 \mathrm{MHz}$ [6], [7] and up to several GHz. As a result, and regarding frequency bands allowed for UWB communication, UWB has, inter alia, the advantage of considerably improving the conditions for an effective radio transmission in tunnels.

In this paper, UWB deterministic channel modeling and characterization in tunnels are mainly discussed. Furthermore, UWB transmission optimized through TimeReversal (TR) technique [8], [9], [10] is investigated and proved to be advantageous in such applications and environments. This is especially the case in terms of increasing Signal-to-Noise Ratio (SNR) and therefore the communication range. This reduces interference effects, receivers complexity, improves data-rates, multi-user capacity and provides Low Probability of Interception (LPI) to non-intentional users.

This paper is organized as follows: Section 2 introduces an overview of the proposed CBTC system. Section 3 describes the UWB channel model characteristics in tunnels and section 4 demonstrates, by means of simulation, the usefulness and effectiveness of UWB-TR combination. Finally, appropriate conclusions are drawn in section 5.

\section{Overview Of The Proposed CBTC System}

In this work, we have chosen to explore the UWB Impulse Radio (UWB-IR) technology. The main objectives 
are to maintain a high speed, robust and highly available train-to-wayside communication by means of UWB transmission in tunnels. The proposed system architecture is illustrated in Figure 1. Ideally, if the communication range

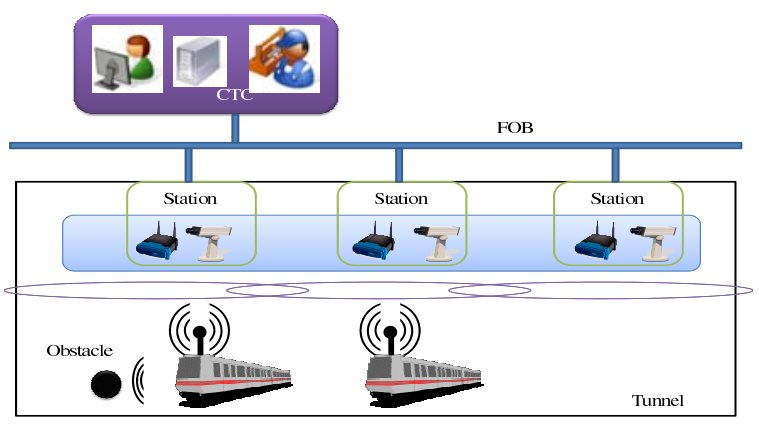

Figure 1. Proposed CBTC system.

is sufficient, access points (APs) equipped with UWBIR modems are deployed along the tunnel at intervals of an inter-station distance, along the transport network. Each AP covers a separate communication area which allows wireless communication, through antennas, with the embedded equipment on the trains circulating in that particular area. Wireless communication equipment is connected to a fiber optic backbone (FOB) insuring a continuous link between ground units and with the CTC. Although this paper concentrates on train-to-wayside communication, UWB radio technology also offers more potential for the control and command of trains:

- It provides potentially high transmission data-rate, using a very large bandwidth;

- It offers high resolution train location because of the fine temporal resolution of the transmitted pulses;

- It adds ability to detect obstacles (radar) due to the impulsive nature of the signals. This capability is essential to avoid obstacles in front of the train or to detect preceding trains;

- Availability and robustness to multi-path are inherent to the large frequency bandwidth;

- UWB CBTC has a low probability of interception to non-intentional receivers due to very low transmitted power spectral densities (PSD). This property is interesting for the safe operation of railway systems;

- It can coexist with other radio systems thanks to low PSD, resulting in fewer requirements of allocated frequencies.

The ability to combine all these assets is a major element of integration that makes UWB a novel, effective and complete solution for a CBTC application [11].

\section{UWB CHANNEL IN TUNNELS}

\section{A. Channel Modeling}

In the early 1970s, Mahmoud and Wait derived a geometrical ray model for straight rectangular tunnels [12]. In the following study, we used the same model. Based on geometrical optics and the ray-tracing techniques, a straight $8 \mathrm{~m}$ wide and $6 \mathrm{~m}$ high, rectangular, infinite tunnel is simulated [10]. Uniform cross-section and lossy, smooth walls are assumed. Regarding this geometry and tunnel cross section, very large relative to our studied wavelengths (10 to $3 \mathrm{~cm}$ ), this deterministic approach seems accurate and easy to implement. Thus, the choice of this approach is suitable compared to the experimental approach which is accurate and reliable but relatively long and complex. The model is exploited in frequency domain, over the 3.1 to $10.6 \mathrm{GHz} \mathrm{FCC}^{2}$ authorized band. The time domain channel impulse response (CIR) is obtained, applying Inverse Fast Fourier Transform (IFFT) algorithm on the total received electric field, for a given $T_{x}-R_{x}$ link, (1):

$$
\begin{aligned}
& E_{\text {tot }}=\sum_{[\text {fmin,fmax }]} E_{0} \frac{\lambda}{4 \pi} \sum_{n=0}^{N-1} \frac{e^{-j k d_{n}}}{d_{n}} R_{V}^{\alpha} R_{H}^{\beta} \\
& h(t)=\mathcal{F}^{-1}\left\{\frac{E_{t o t}}{E_{0}}\right\}
\end{aligned}
$$

where $E_{0}$ denotes the reference electric field, $N$ is the considered images of source $T_{x}, k$ is the wave number, $R_{V(H)}$ and $\alpha(\beta)$ are, respectively, the complex reflection coefficient and the number of reflections on the vertical(horizontal) walls and $d_{n}$ is the length of the $n^{t h}$ ray. The model assumes non-magnetic and homogeneous interfaces (same material), so the relative complex permittivity is only frequency-dependent and permeability $\mu_{r}=1$. Table I summarizes the channel parameters used for this evaluation.

TABLE I.

CHANNEL MODEL PARAMETERS.

\begin{tabular}{|c|c|}
\hline \hline Parameter & Value \\
\hline Link & LOS \\
Frequency band [GHz] & $3.1-10.6(1601 p t s)$ \\
Conductivity $[\mathrm{S} / \mathrm{m}]$ & $\sigma=0.05$ \\
Permeability $[\mathrm{H} / \mathrm{m}]$ & $\mu_{r}=1$ \\
Permittivity [F/m] & $\epsilon_{r}=7,\left(\underline{\epsilon}_{r} \approx \epsilon_{r}-j 60 \sigma \lambda\right)$ \\
Tunnel size (hxw) $[\mathrm{m}]$ & $6 \times 8$ \\
Nb of reflections & $\alpha_{\max }=12$ \\
$\mathrm{~N}$ (virtual sources) & $168+$ direct path \\
\hline \hline
\end{tabular}

\section{B. Channel Characterization}

To perform the channel characterization, we used the theoretical model and we consider the simulation setup shown in Fig. 2. The transmitter is located at a particular place along the main axis of the tunnel, at different lateral positions $\left(P_{1}, P_{2}, P_{3}, P_{4}\right)$, according to the parameter to be estimated. The receiver moves along the main axis of the tunnel from a reference distance $(1 \mathrm{~m})$ then starting from $5 \mathrm{~m}$ up to a given distance $d_{n}$, using a $5 \mathrm{~m}$ step. To optimize this presentation, we first present the channel estimated parameters in the $3-10 \mathrm{GHz}$ frequency band. Then, at the end of this section, we give the results of the same analysis applied to two reduced frequency bands (2-6 GHz and 6-10 GHz).

\footnotetext{
${ }^{2}$ Federal Communications Commission
} 

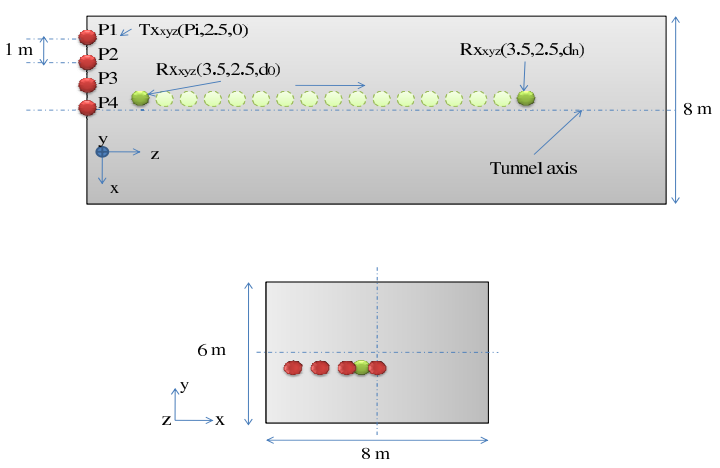

Figure 2. Simulation setup.

As an initial step, frequency responses and time impulse responses were computed. Fig. 3 shows transfer functions obtained for $R_{x}$ locations up to $d_{n}=55 \mathrm{~m}$. The

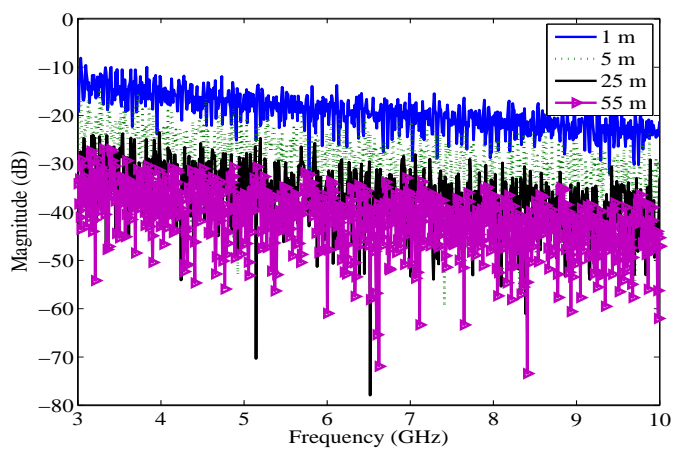

Figure 3. UWB frequency responses $(1-55 \mathrm{~m})$.

corresponding time domain impulse responses, averaged and normalized power delay profile (PDP) are given, respectively, in Fig. 4 and in Fig. 5. Next, these results are used to determine other parameters, such as delay spread, coherence bandwidth and tunnel path loss, which are now analyzed.

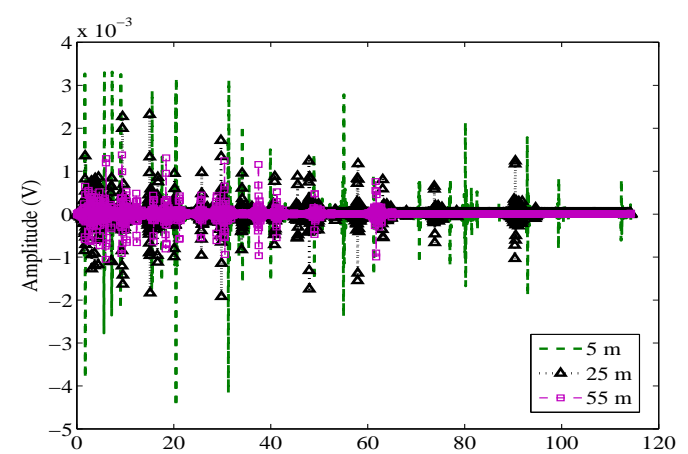

Figure 4. Corresponding impulse responses (1-55 m).

1) RMS Delay Spread $\tau_{R M S}: \tau_{R M S}$ is an important parameter which provides information to evaluate the level of the inter-symbol interference (ISI) in digital communications. $\tau_{R M S}$ was computed from the PDP of

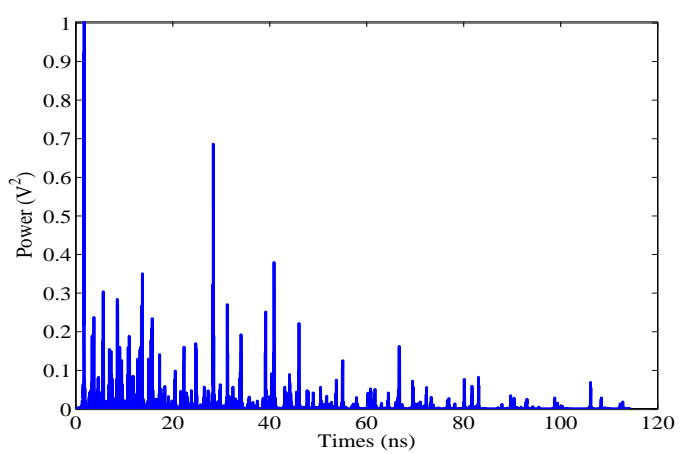

Figure 5. Averaged and normalized power delay profile (PDP).

Fig. 5. We found that this parameter varies around a mean value of $25 \mathrm{~ns}$.

a) Distance-Dependent RMS Delay Spread: To evaluate the distance dependence of $\tau_{R M S}$, we used the four configurations of $T_{x}$ antenna represented in Fig. 2. The curves in Fig. 6 represent $\tau_{R M S}$ variation according to distance. From these curves, the estimated $\tau_{R M S}$ varies

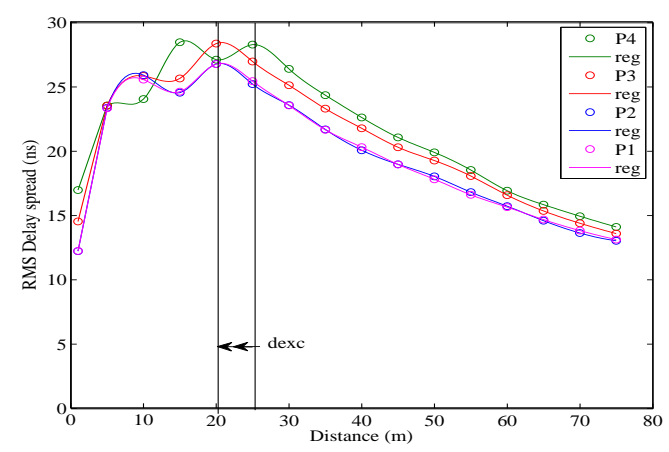

Figure 6. $\tau_{R M S}$ variation according to $T_{x}-R_{x}$ separation distance.

between 12 and $30 \mathrm{~ns}$. For short $R_{x}$ to $T_{x}$ distances, we note an important dispersive behavior of the channel (increase and fluctuation of $\tau_{R M S}$ values). Then, starting from about 20-25 m, the channel becomes less dispersive ( $\tau_{R M S}$ falls and stabilizes).

A similar behavior was observed in [13]. A study was conducted by the latter to explain such an evolution of $\tau_{R M S}$ according to distance. The explanation lies in the existence of two distinct areas with different behaviors in the tunnel: the excitation zone of the tunnel and the zone beyond. The excitation zone was defined in [14]. In that zone, transfer of radiofrequency energy occurs between the transmitter and the tunnel and, considering the raytheory, many different radio paths coexist. Beyond that zone, many radio paths are already severely attenuated. Therefore, the highest values of $\tau_{R M S}$ were observed in the excitation zone, with irregular variations. Beyond this zone, $\tau_{R M S}$ decreases before stabilizing. Thus, $\tau_{R M S}$ depends on $d_{\text {exc }}$.

Remarks: This result leads to interesting remarks in relation with our application. In fact, the maximum data- 
rate which may be sent before the time dispersion produces significant errors from inter-symbol interference in the channel is related to $\tau_{R M S}$ by (2) [15]:

$$
D R_{\max }=\frac{1}{10 \tau_{R M S}}
$$

Now, the results show that $\tau_{R M S}$ values do not exceed $30 \mathrm{~ns}$. Considering the average value $(25 \mathrm{~ns})$, it is possible to ensure a maximum equivalent data-rate $D R=1 /\left(10 \tau_{R M S}\right)=4 \mathrm{Mbps}$ to avoid ISI without special precaution. In order to achieve a greater throughput, this excitation zone has to be carefully explored, since the maximum throughputs we can ensure within and outside this zone will be different. Indeed, $\tau_{R M S}$ changes according to $d_{\text {exc }}$ (increases before $d_{\text {exc }}$ and decreases after), so we should:

- keep an inter-APs distance greater than $d_{\text {exc }}$ to avoid dispersive behavior of the channel and to allow the maximum throughput,

- otherwise, use techniques like time-reversal to improve the results at shorter distances $\left(d<d_{e x c}\right)$

Taking this initial example, a data-rate $D R \approx 7 \mathrm{Mbps}$ $\left(\tau_{R M S} \approx 14.5 \mathrm{~ns}\right)$ could be achieved without equalization at a range of $75 \mathrm{~m}$. It should be noted that the type of antenna used also influences the time dispersive properties of the channel. Indeed, directional antennas reduce the channel delay spread which results in ISI reduction and, in consequence, throughput improvement [16].

2) Coherence Bandwidth $B_{c}$ : This parameter is also an important indicator of the channel frequency selectivity. It is defined as the frequency range over which the channel can be considered "flat" and it can be computed from $\tau_{R M S}$ by (3) or (4):

$$
\begin{aligned}
& B_{c 50 \%}=\frac{1}{5 \tau_{R M S}} \\
& B_{c 90 \%}=\frac{1}{50 \tau_{R M S}}
\end{aligned}
$$

The obtained value of $B_{c 50 \%}$ for the $3-10 \mathrm{GHz}$ frequency band is $8.12 \mathrm{MHz}$. Compared to UWB signals bandwidth, this value confirms the frequency-selective fading nature of the channel, which means that different frequency components of the signal will experience decorrelated fading.

3) Ray and Cluster Exponential Decay Factors: In the presence of the clustering phenomenon in the UWB channel, most previous works assume an exponential decay for both cluster power and ray power within a cluster as a function of the delay [17]. Two parameters $\Gamma$ and $\gamma$ characterize, respectively, the inter- and intra-cluster decaying. The mean values for these two parameters were obtained by linear regression from the PDP. The obtained values of $\gamma$ and $\Gamma$ were estimated around $7 \mathrm{~ns}$ and $30 \mathrm{~ns}$ respectively (see the following table II).

4) Path Loss: a) Tunnel Path Loss: Previous studies on path loss along Line of Sight (LOS) have demonstrated the existence of two distinct regions, each one presenting its own attenuation factor. In urban and rural environments, these two regions have quite different characteristics and are separated by a break point $d_{c}$. Before the break point (near region), propagation loss is low (path loss exponent $n_{d}=2$ ) and after the break point (far region), path loss exponent increases $\left(n_{d}>2\right)$. This break point distance can be expressed in terms of transmit and receive antenna heights and the wavelength [18].

Unlike previous open-area environments, the opposite occurs in tunnel environments in terms of propagation loss. Indeed, propagation loss is more significant in the near region than in the far one. Ref. [19] showed that the path loss in tunnel follows two models:

- one for distances close to the transmit antenna, similar to free-space path loss $\left(n_{d}=2, d<d_{c}\right)$,

- and another one starting from dc. For these distances, the waveguide effect takes place and $n_{d}<2$.

In the near region, the free-space model can be explained by considering that propagation occurs in the Fresnel zone of the transmit antenna. After the break point, constructive reflections dominate, resulting in loss diminution. This waveguide effect then extends over long distances. This also explains the results found in (IIIB.1.a) concerning the decrease in the time dispersive behavior of the channel at long distances.

b) Distance-Dependent Path Loss: To evaluate distance-dependent path loss, we now consider communication ranges of 1 to $600 \mathrm{~m}$. The curves in Fig. 7 represent propagation path loss results obtained by averaging losses over the entire frequency band for each $T_{x}-R_{x}$ distance (5):

$$
P L(d)=\frac{1}{M N} \Sigma_{i=1}^{N} \Sigma_{j=1}^{M}\left|H\left(f_{i}, t_{j}, d\right)\right|^{2} \propto d^{n_{d}}
$$

where $N$ is the total number of frequency points over bandwidth, $M$ is the total number of frequency snapshots over time, and $n_{d}$ represents the path loss exponent. To

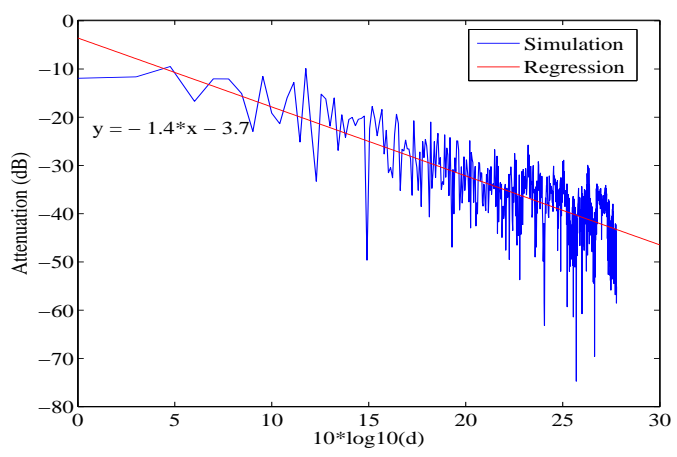

Figure 7. Ray-tracing computed UWB path loss in tunnel between 1 and $600 \mathrm{~m}$.

determine $n_{d}$ we proceed by linear regression on the path 
loss expressed in $\mathrm{dB}$ by (6):

$$
P L(d)_{d B}=P L_{0}+10 n_{d} \log _{10}\left(\frac{d}{1 m}\right)+\chi_{\sigma}
$$

$P L_{0}$ represents the free-space path loss in the far field of the antennas at $1 \mathrm{~m}$ and $\chi_{\sigma}$ represents the lognormal shadowing. Over the considered frequency band, the obtained mean value of the path loss exponent is $n_{d}=1.4$.

Comparison with narrow band results: UWB path loss results were compared to those of narrow band results. $n_{d}$ was estimated for frequencies between 2 and $10 \mathrm{GHz}$ and the same 1 to $600 \mathrm{~m}$ ranges. The results obtained and their approximation by linear regression are presented in Fig. 8. Narrow band $n_{d}$ is estimated around

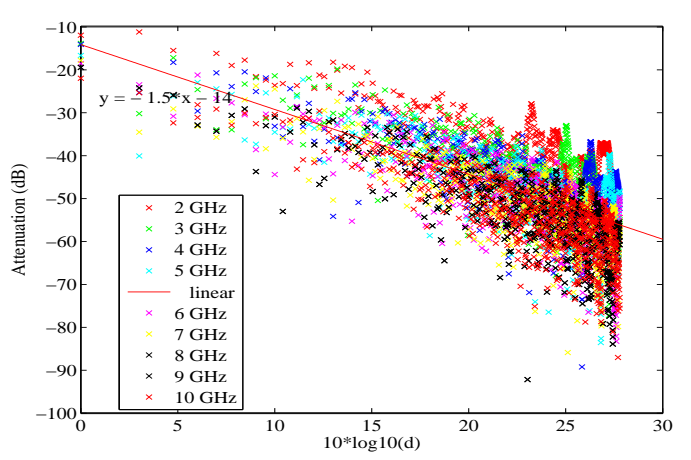

Figure 8. Ray-tracing simulated narrowband path loss in tunnel between 1 and $600 \mathrm{~m}$.

1.63 and its variation according to frequency is given in Fig. 9.

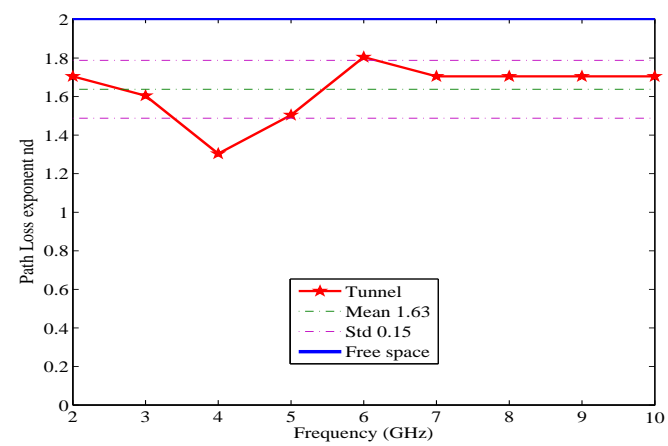

Figure 9. Frequency impact on path loss exponent $n_{d}$.

Determination of the propagation regions: To identify the propagation regions, we used the method described in [19]. Results in the $3-10 \mathrm{GHz}$ band give $190 \mathrm{~m}<$ $d_{c}<254 \mathrm{~m}$.

c) Frequency-Dependent Path Loss: The frequency dependency of UWB path loss can be modeled using two models:

- the model adopted in the IEEE.802.15.4a standard channel defined in [20]:

$$
\sqrt{P L(f)} \propto f^{-n_{f 1}}
$$

where, $n_{f 1}$ represents the frequency decaying factor. It generally varies between 0.8 and 1.4 .

- and the exponential model [21] given by:

$$
P L(f) \propto k . e^{-n_{f 2} f}
$$

where $n_{f 2}$ varies from 1 to 1.4 .

The frequency decaying factors were calculated using least-square curve fittings on (7) and (8). The values of $n_{f 1}$ and $n_{f 2}$ are reported in table II.

Summary: As said before, the same analysis was applied for the 2-6 GHz and 6-10 GHz frequency bands. Results are summarized in the following table.

TABLE II.

SUMMARY OF UWB CHANNEL CHARACTERISTICS IN TUNNEL.

\begin{tabular}{|c|c|c|c|}
\hline \hline Frequency band [GHz] & $3-10$ & $2-6$ & $6-10$ \\
\hline Range d [m] & $1-55$ & $1-55$ & $1-55$ \\
\hline Path Loss & \multicolumn{3}{|l|}{} \\
\hline$P L_{0}[\mathrm{~dB}]$ & 19.45 & 183 & 22.63 \\
Mean Attenuation [dB] & 35.48 & 34.54 & 38.64 \\
$n_{d}$ & 1.4 & 1.5 & 1.2 \\
$n_{f 1}$ & 1 & 0.95 & 1.05 \\
$n_{f 2}$ & 1.2 & 1.4 & 1.2 \\
\hline PDP & \multicolumn{3}{|l}{} \\
\hline$\tau_{R M S}[\mathrm{~ns}]$ & 24.63 & 27.20 & 26.75 \\
$B_{c 50 \%}[\mathrm{MHz}]$ & 8.12 & 7.35 & 7.47 \\
$\gamma($ mean) $[\mathrm{ns}]$ & 7 & 6 & 8 \\
$\Gamma$ (mean) $[\mathrm{ns}]$ & 30 & 26 & 23 \\
\hline \hline
\end{tabular}

We note a lower value of parameter $n_{d}$ in the 6$10 \mathrm{GHz}$ higher frequency band, compared to the $2-6 \mathrm{GHz}$ lower band: $n_{d_{(6-10 G H z)}}=1.2, n_{d_{(2-6 G H z)}}=1.5$. All these values of $n_{d}$ are below those corresponding to free-space propagation $\left(n_{d}=2\right)$. A difference of about $4 \mathrm{~dB}$ is also observed, on the mean value of attenuation between the lower and the higher bands. This means that the higher band suffers less attenuation than the lower band. In tunnels, longitudinal attenuation decreases as frequency increases but we have also to recall that our model considers smooth walls. Taking into account tunnel wall roughness, this conclusion could be modified.

According to these results, we conclude that $\tau_{R M S}$ is not influenced by the frequency. This observation was also noticed in several studies [22], [23].

\section{TIME-REVERSAL EVAluATION}

\section{A. Time Reversal}

In spite of the very high time resolution of UWBIR signals making the probability of fading very weak, the process of collecting energy that is dispersed in rich multi-path environments seems to be a very difficult task [24], [25]. Moreover, given the stringent regulation constraints on power, receivers must be very efficient at gathering up the multi-path energy. One way is to use complex systems such as RAKE receivers, using a large number of fingers, or Multiple Input Multiple Output (MIMO) systems. Recently, TR prefiltering has emerged as a solution to reduce this complexity, thus preserving the important low-cost advantage of UWB. The TR technique, originating from underwater acoustics [26], works as follows: 
Considering an UWB communication link between a transmitter noted $\mathrm{A}$ and a receiver noted $\mathrm{B}$, the complex channel impulse response (CIR) is first estimated and recorded at source $\mathrm{A}$. Then, data is prefiltered through a time-reversal filter, having the same previously recorded and time-reversed CIR as the impulse response. Finally, the resulting output is sent over the propagation channel to $\mathrm{B}(9)$.

$$
s_{B}=s_{A} \otimes C I R_{A B}^{*}(-t) \otimes C I R_{A B}(t)
$$

where $s_{A}$ and $s_{B}$ are respectively the transmitted and received signals. $\otimes$ denotes convolution and $C I R_{A B}^{*}(-t)$ represents the time-reversed and complex-conjugated CIR. Thus, (10) gives the new equivalent CIR as:

$$
h e q(t)=C I R_{A B}^{*}(-t) \otimes C I R_{A B}(t)=R_{A B}^{a u t o}(t)
$$

where $R_{A B}^{\text {auto }}(t)$ indicates the channel autocorrelation function. This has, as a direct consequence, the concentration in time and space of the received energy. This focusing process appears to be very beneficial to UWB communication [27], [28], increasing SNR and, therefore, the communication range, reducing interference effects (ISI, $\mathrm{MUI}^{3}$ ), receivers complexity, as well as improving data-rates, multi-user capacity and providing LPI. In this paper we assume the Channel State Information (CSI) to be perfectly known at the transmitter and that the channel remains stationary during the two successive stages of channel estimation and data transmission.

\section{B. TR simulations}

Under these assumptions, our simulations propose to demonstrate the benefit of UWB-TR transmission in the tunnel, especially in terms of ISI and MUI reduction, as well as providing higher throughputs at extended communication ranges. Both Single Input Single Output (SISO) and Multiple Input Single Output (MISO) transmission were tested for both the conventional transmission mode (without TR) and the TR one.

1) Temporal focusing: The first demonstration proposes to evaluate temporal compression for three different $T_{x}-R_{x}$ separations, $d=5 \mathrm{~m}$. Then, $d=50 \mathrm{~m}$ and, finally, $d=100 \mathrm{~m}$. Four scenarios have been tested:

- SISO without TR,

- SISO with TR,

- MISO, using TR with two transmit antennas $\left(N T_{x}=\right.$ 2),

- MISO-TR with four transmit antennas $\left(N T_{x}=4\right)$.

For SISO configurations, both $T_{x}$ and $R_{x}$ antennas were centered on the tunnel cross section. For MISO situations, as depicted in Fig. 10, $R_{x}$ antenna is centered, $T_{x}$ antennas are positioned at the corners of a $50 \mathrm{~cm}$ square, one corner being the center of the tunnel.

To evaluate the temporal focusing effectiveness, we compute the $R M S$ delay spread using non-TR and TR configurations (see table III). As demonstrated in [29] for residential indoor environments case, the predicted delay

${ }^{3}$ Multi-User Interference
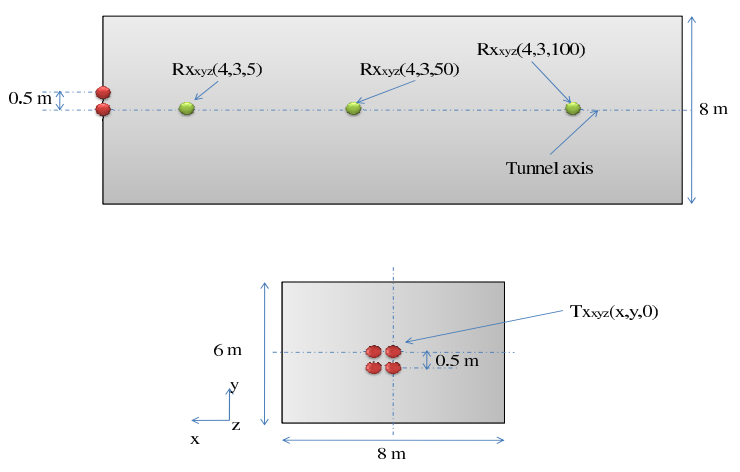

Figure 10. Time focusing simulation setup.

TABLE III.

\begin{tabular}{|c|c|c|c|c|c|}
\hline & $5 \mathrm{~m}$ & $50 \mathrm{~m}$ & $100 \mathrm{~m}$ \\
\hline \multicolumn{3}{|c|}{ Without Time-Reversal } & $31.55 \mathrm{~ns}$ & $13.93 \mathrm{~ns}$ & $8.20 \mathrm{~ns}$ \\
\hline \multirow{3}{*}{ 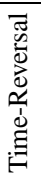 } & \multicolumn{2}{|c|}{ SISO } & $32.55 \mathrm{~ns}$ & $13.95 \mathrm{~ns}$ & $8.42 \mathrm{~ns}$ \\
\hline & \multirow{2}{*}{ MISO } & $N T_{x}=2$ & $19.82 \mathrm{~ns}$ & $12.08 \mathrm{~ns}$ & $7.67 \mathrm{~ns}$ \\
\hline & & $N T_{x}=4$ & $16.31 \mathrm{~ns}$ & $10.62 \mathrm{~ns}$ & $7.47 \mathrm{~ns}$ \\
\hline
\end{tabular}

RMS DELAY SPREAD COMPARISON.

spread in the tunnel environment for the SISO-TR case is not reduced but increased. This results in the presence of temporal sidelobes, referring to the residual portion of the autocorrelation. However, the response is symmetric and almost all the energy is concentrated around $\mathrm{t}=0$ resulting in a clear signal peak, that is several $\mathrm{dB}$ above the temporal sidelobes. In the case of MISO-TR, temporal focusing is clearly improved. Indeed, MISO-TR acts as a technique that maximizes the desired received signal energy to temporal sidelobe energy ratio. Results also show that increasing the number of transmitting antennas involves a better temporal compression, especially at the shortest $T_{x}-R_{x}$ distances (by about a factor of 0.5). As explained before, this can be explained by the scattering effect, more important at shortest distances than at farther distances. Consequently, the transmitter correlations are lower, resulting in a better temporal compression. This observation confirms that the TR technique is quite effective in high scattering tunnel environments, especially at short ranges. Moreover, since channel delay spread is significantly reduced by using MISO-TR, ISI can also be significantly reduced. Consequently, higher throughput can be obtained. In our situation, according to CBTC requirements a few Mbps are enough to provide all the required communication resources. Moreover, the wave-guide effect of the tunnel keeps energy inside the dedicated transport environment. Considering the average values of $\tau_{R M S}$ in these ranges, we deduce that the maximum data-rate can be improved from 5.5 Mbps (without TR) to $8.3 \mathrm{Mbps}$ (with TR $N T_{x}=4$ ).

2) Spatial focusing: To evaluate the spatial focusing, we chose a reference $T_{x}-R_{x}$ pair. Then, we considered an area around the intended receiver $R_{x}$, as shown in 
Fig. 11. $\lambda$ is the mean wavelength in the swept frequency band. Using the new equivalent time reversed CIRs, the
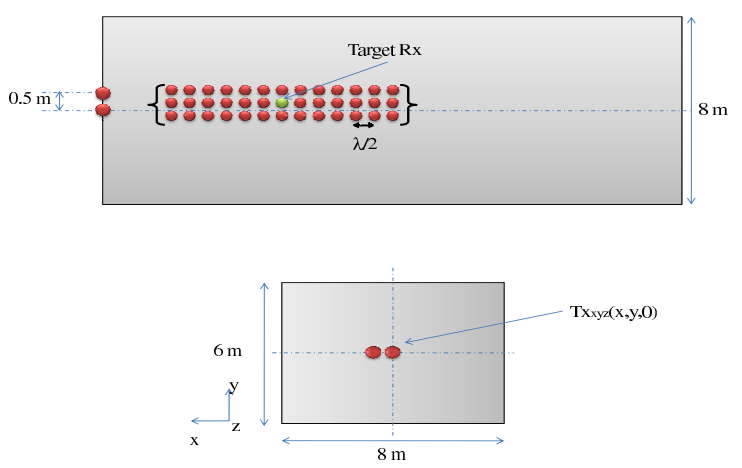

Figure 11. Spatial focusing simulation setup.

spatial focusing gain is defined as the ratio between the maximum power at reference location $r_{0}$, where the target receiver is located, to the maximum power at a given location $r=r_{0}+\delta x$ away from the target (11):

$$
\begin{aligned}
s g_{d B} & =\max \left\{P\left(r_{0}\right)\right\}-\max \{P(r)\} \\
& =\max \left\{\left|R^{\text {auto }}\left(r_{0}\right)\right|^{2}\right\}-\max \left\{\left|R^{\text {cross }}\left(r, r_{0}\right)\right|^{2}\right\}
\end{aligned}
$$

Low spatial focusing gain at distance $r$ away from the intended receiver indicates that a nearby receiver at that location would not be able to intercept the communication successfully. Spatial focusing increases the system security and CBTC effectiveness by addressing different trains in the tunnel selectively. This will also enhance multi-user system capacity in the tunnel.

Two configurations were investigated for a $T_{x}-R_{x}$ separation of $d=5 \mathrm{~m}$ : SISO-TR and MISO-TR (with $N T_{x}=2$ ). Fig. 12 illustrates power normalized to peak received in the area without $\mathrm{TR}$. We observe that all users

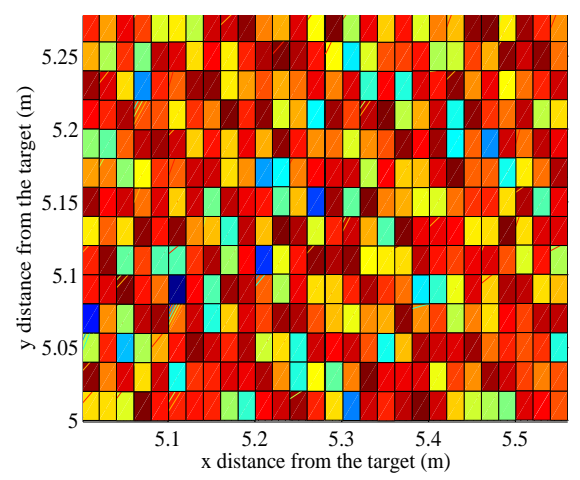

Figure 12. Power spatial repartition at the reception.

in this area receive approximately the same power level. This means that the communication can be intercepted by any receiver and, in our case, the MUI problem can really affect the multiple access performance. By using TR (Fig. 13), the power is decaying rapidly when moving away from the target. Several situations of the reference target were tested in the virtual grid and the results were tested very close. The power is at least $3 \mathrm{~dB}$ lower inside $\pm \lambda / 2$ interval, and lower values are observed beyond this interval.

Next, we considered the case of multiple transmit antennas $\left(N T_{x}=2\right)$. Fig. 14 illustrates a realization comparing SISO-TR (Fig. 14(a)) and MISO-TR (Fig. 14(b)) for the same configuration of the target receiver. We obtained that MISO-TR performs better spatial focusing around the target. Of course, results are better as transmitters are decorrelated. A measurement campaign was also conducted to validate these results. Corresponding results are reported in [10].

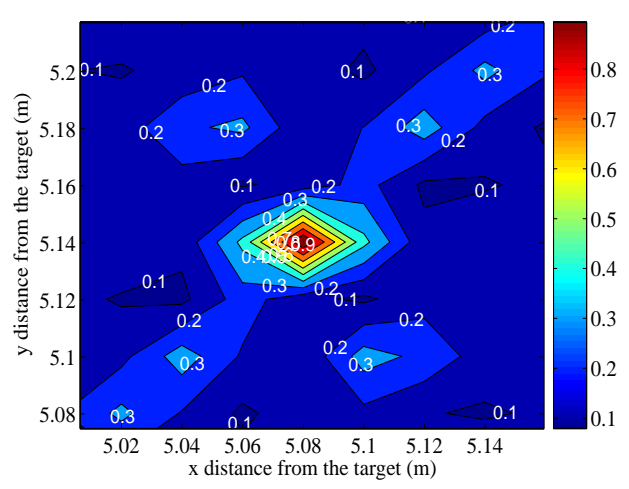

Figure 13. Spatial focusing gain in SISO-TR situation.

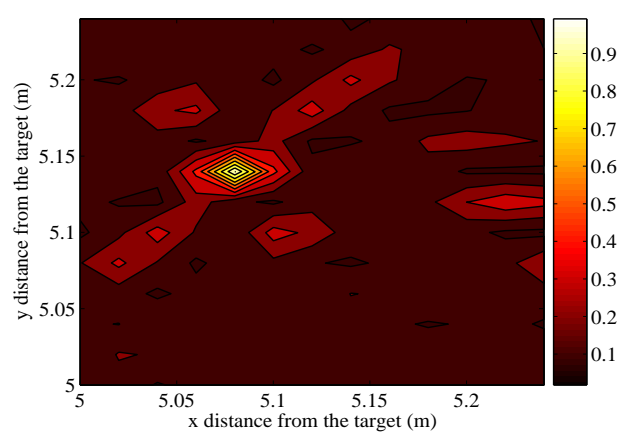

(a) SISO-TR

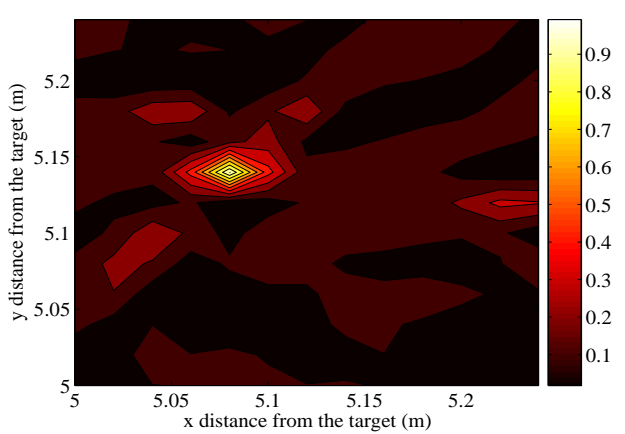

(b) MISO-TR $\left(N T_{x}=2\right)$

Figure 14. Spatial focusing gain comparison between SISO and MISO.

\section{CONCLUSiON}

In this paper, a new CBTC scheme based on UWB-IR radio propagation in tunnels was proposed. A ray-based 
channel model in tunnels was presented and characterized. Among other parameters channel path loss and RMS delay spread and their frequency and distance dependencies were investigated.

UWB associated to Time-Reversal constitutes a good technical combination to achieve reliable communication in railway tunnels. We have shown how TR can help to simplify signal detection without using complex algorithms at the receiver level. The results obtained prove that temporal and spatial focusing can be improved by using multiple antennas at the transmitter. Thus, multi-user system capacity and data-rate can be greatly increased.

In the real world of mobile train-to-wayside and trainto-train communication, the task of channel estimating required by TR will be an obstacle. However, deterministic propagation models exist that can run in real time, and guided trains always use the same trajectories. Thus, considering that lateral positions of the antenna will be almost constant on board the train, and that UWB provides accurate $T_{x}$ to $R_{x}$ distance measurements, we think that this challenge can be addressed. Current work focuses on evaluating UWB-TR transmission by means of real-time measurements on a real vaulted tunnel using appropriate equipment.

\section{REFERENCES}

[1] M. Fitzmaurice, "Use of $2.4 \mathrm{GHz}$ Frequency Band for CBTC Data Communication Systems," in Joint Rail Conference. Parson Corporation (USA), March 2006.

[2] E. Kuun, "Open Standards for CBTC and CCTV RadioBased Communication," Alcatel Canada, APTA Rail Transit Conference Proceedings, June 2004.

[3] Official IEEE 802.11 Working Group Project Timelines, [Online] Available: http://grouper.ieee.org/ groups/802/11/Reports/802.11_Timelines. htm.

[4] Rail Transit Vehicle Interface Standards Committee, [Online] Available: http://grouper.ieee.org/ groups/railtransit/

[5] T. Sullivan, "The IEEE 1473-L Communications Protocol: Experience in Rail Transit," On-board ITS in rail transit, track 5 - technical forums, June 2002.

[6] R. Klingler, "Radio Coverage for Road and Rail Tunnels in 75 to $1000 \mathrm{MHz}$," in IEEE $41^{\text {st }}$ Veh. Technol. Conf., pp. 433-438, 1991.

[7] Y. P. Zhang and H. J. Hong, "Ray-Optical Modelling of Simulcast Radio Propagation Channels in Tunnels," IEEE Trans. Veh. Tech., vol.53, pp.1800-1808, November 2004.

[8] S. M. Emami et al., "Predicted Time Reversal Performance in Wireless Communications Using Channel Measurements," IEEE Comm. Lett., 2004.

[9] G. Lerosey, "Time Reversal of Electromagnetic Waves," Phys. Rev. Lett. p. 194301, 922004.

[10] H. Saghir et al., "Train-to-Wayside Wireless Communication in Tunnel Using Ultra-Wideband and Time Reversal," Transportation Research, Part C: Emerging Technologies, vol. 17, Issue 1, pp. 81-97, February 2009.

[11] M. Heddebaut, A. Rivenq-Menhaj, J-M. Rouvaen, F. Elbahhar, and J-P. Ghys, "Device and Method for Positioning and Controlling Railway Vehicles with Ultra-Large Bandwidth," US Patent and Trademark Office Application \#: 20060151672, 2006.
[12] S. F. Mahmoud and J. R. Wait, "Geometrical Optical Approach for Electromagnetic Wave Propagation in Rectangular Mine Tunnels," Proc. Radio Science, no. 9, pp. 1147-1158, 1974.

[13] F. M. Pallares, F. J. P. Juan, and L. Juan-Llacer, "Analysis of Path Loss and Delay Spread at $900 \mathrm{MHz}$ and $2.1 \mathrm{GHz}$ while Entering Tunnels," IEEE Trans. on Veh. Tech., vol. 50, No. 3, pp. 767-776, May 2001.

[14] P. Mariage, M. Lienard, and P. Degauque, "Theoretical and Experimental Approach of the Propagation of High Frequency Waves in Road Tunnels," IEEE Trans. Ant. Propagat., vol. 42, pp. 7581, January 1994.

[15] T. S. Rappaport, K. Blankenship, and H. Xu, "Propagation and Radio System Design Issues in Mobile Radio Systems for the GloMo Project," [Online] Available: http: //www.mprg.ee.vt.edu/research/ glomo/prev2.html, 1997.

[16] J. A. Dabin et al., "The Effects of Antenna Directivity on Path Loss and Multipath Propagation in UWB Indoor Wireless Channels," IEEE Conference on Ultra Wideband Systems and Technologies, vol., no. 16-19, pp. 305-309, November 2003.

[17] A. F. Molisch, "Ultrawideband Propagation ChannelsTheory, Measurement, and Modeling," IEEE Trans. Veh. Tech., vol. 54, no. 5, pp. 1528-1545, 2005.

[18] H. H. Xia, H. L. Bertoni, L. R. Maciel, A. L. Stewart, and R. Rowe, "Radio Propagation Characteristics for Line-ofSight Microcellular and Personal Communications," IEEE Trans. Ant. Propagat., vol. 41, pp. 1439-1447, October 1993.

[19] Y. P. Zhang, "Novel Model for Propagation Loss Prediction in Tunnels," IEEE, Trans. Veh. Tech., vol. 52, No. 5, pp. 1308-1314, September 2003.

[20] J. Kunisch and J. Pamp, "Measurement Results and Modeling Aspects for the UWB Radio Channel," IEEE Conference on Ultra Wideband Systems and Technologies, pp. 19-23, May 2002.

[21] A. Alvarez, G. Valera, M. Lobeira, R. Torres, and J. L. Garcia, "New Channel Impulse Response Model for UWB Indoor System Simulations," Proc. VTC Spring 2003, pp. 1-5, Seoul, Korea, April 2003.

[22] T. Jamsa, V. Hovinen, and L. Hentil, "Comparison of Wideband and Ultra-Wideband Channel Measurements," in COST 273, Gothenburg, Sweden, June 2004.

[23] P. Pagani, "Caractérisation et Modélisation du Canal de Propagation Radio en Contexte Ultra Large Bande," PhD. dissertation (in French), Institut National des Sciences Appliquées de Rennes, France, November 2005.

[24] M. Z. Win and R. A. Scholtz, "On the Robustness of Ultra-Wide Bandwidth Signals in Dense Multipath Environments," IEEE Comm. Lett., vol. 2, no. 2, pp. 51-53, February. 1998.

[25] M. Z. Win and R. A. Scholtz, "On the Energy Capture of Ultra-Wide Bandwidth Signals in Dense Multipath Environments," IEEE Comm. Lett., vol. 2, no. 9, pp. 245247, September 1998.

[26] G. F. Edelmann et al., "An Initial Demonstration of Underwater Acoustic Communication Using Time Reversal," IEEE J. Ocean. Eng., vol. 27, pp. 602-609, 2002.

[27] R. C. Qiu, C. Zhou, N. Guo, and J. Zhang, "Time Reversal with MISO for Ultra-Wideband Communications: Experimental Results," IEEE Antenna and Wireless Propagation Letters, vol. 5, pp. 269-273, 2006.

[28] T. Strohmer, M. Emami, J. Hansen, G. Papanicolaou, and P. Arogyaswami, "Application of Time-Reversal with MMSE Equalizer to UWB Communications," in Proc. IEEE Global Telecommunications Conference, vol. 5, pp. 3123-3127, December 2004.

[29] P. Kyritsi and G. Papanicolaou, "One-Bit Time Reversal for WLAN Applications,” vol. 1, pp. 532-536, 2005. 
Hassane SAGHIR was born in Tiznit, Morocco, in 1980. He received his M.S. degree in Telecommunication and signal processing from the University of Valenciennes, France, in 2005.

$\mathrm{He}$ is currently a Ph.D. candidate at the University of Valenciennes, France. His current research interests include railway transportation systems, and ultra wideband technology.

Marc Heddebaut was born in Somain, France, in 1955. He received the M.S. and Ph.D. degrees in electronics from the University of Lille France in 1980 and 1983, respectively. He joined the French National Institute for Transportation and Safety Research (INRETS) in 1983 and became a senior researcher in 1988. Since 1979, he has been working in the field of land mobile communication and electromagnetic compatibility. His primary interests are telecommunication systems dedicated to land transport, mobile localization and command control of automated vehicles.

Fouzia Elbahhar was born in 1975. She received the M.S. and $\mathrm{Ph} . \mathrm{D}$. degrees from the University of Valenciennes (France) in 2000 and 2004, respectively. She is actually employed as Researcher at INRETS/LEOST, Villeneuve d'Ascq, France. She is especially involved in Ultra WideBand technology. Her primary interest is in signal processing applied to intelligent transports application especially in communications and localization.

Atika Rivenq Professor and member of ComNum Group. She was graduated Engineer from The ENSIMEV School in 1993, received the M.S. degree in 1993 and then her Ph.D. degree in 1996 from the University of Valenciennes (France). She is Professor in electronics at this university with the IEMNDOAE Lab. Her main activity is in signal processing applied to intelligent transport systems (localisation, collision avoidance radar, cruise control associated to GPS). She Participates to many national and European projects dedicated to guided-train communication and inter-vehicles communication especially using UWB technology.

Jean Michel Rouvaen was born in Maubeuge, France, in 1947. He received his Ph.D. degree in Electronics from the Universities of Paris VI and Valenciennes, France, in 1976. Currently he is a Professor at the University of Valenciennes, France, where he teaches Electronics, Signal Processing and Telecommunications. Previously, he was involved in ultrasonics and acousto-optics.

His current research interests is focused in: telecommunication systems and their application to security in intelligent transportation systems. 